\title{
Clinical results of continuous flow ventricular assist devices: A comparative analysis between heartmate 3 and heart ware. Are centrifugal pumps all the same?
}

\author{
Anna Nowacka ${ }^{1 *}$, Matthias Kirsch ${ }^{1}$, Jonathan Ruggiero ${ }^{1}, J_{u l i e n}$ Regamey $^{2}$, Roger Hullin $^{2}$, Carlo Marcucci $^{3}$ and Piergiorgio Tozzi $^{1}$ \\ ${ }^{1}$ Department of Cardiac surgery, University Hospital, Lausanne, Switzerland \\ ${ }^{2}$ Department of Cardiology, University Hospital, Lausanne, Switzerland \\ ${ }^{3}$ Department of Anesthesiology, University Hospital, Lausanne, Switzerland
}

\begin{abstract}
Background: Compare clinical outcomes of the last generation centrifugal LVADs implanted as bridge to transplant.

Methods: Retrospective analysis of demographics, clinical parameters, surgical technique, adverse events and survival in patients treated with LVAD centrifugal pump as bridge to transplant. Primary endpoint was survival at one year. Secondary endpoints were device related complications.

Results: Thirty-two (32) adult patients received centrifugal LVAD: 16 (50\%) the HW and 16 (50\%) the HM3. There were no significant differences in survival at 1,3 and 12 months post-implantation. Hemorrhage requiring surgery occurred in 9 (56.3\%) patients in the HW group and 5 (31.3\%) in the HM3 group. Stroke occurred in $2(12 \%) \mathrm{HW}$ and 1 (6.3\%) HM3. Two patients required surgery to treat infection of the driveline, one in each group.
\end{abstract}

Conclusions: Both devices provided excellent hemodynamic support and had similar stroke and bleeding complication rates even if the HM3 was implanted in sicker patients.

\section{Introduction}

End stage heart failure refractory to maximal conventional medical therapy remains one of the most complex therapeutic challenges. The prevalence of the patients with heart failure is approximately $1-2 \%$ of the adult population in developed countries and affects up to $10 \%$ of adults over 70 years old $[1,2]$. This prevalence increases not only with age but also in time. Heidenrich et al. expect a $46 \%$ increase in the total US population suffering heart failure from 2012 to 2030 (from 5.8 million in 2012 to 8.4 million in 2030) [3]. This epidemic explosion is probably due to the increasing age of the population and, to a better management of cardiac pathologies.

Recent improvements in devices' design and technology have led to a reevaluation of the role of ventricular assist devices (VADs) therapy. New generation VADs are centrifugal pumps designed to be more durable, smaller, with optimized blood flow through the pump, decreasing the risk of thrombosis and hemolysis.

The ENDURANCE study [4] did not demonstrate the superiority of the HeartWare device over the HeartMate II device, while the Momentum 3 [5] study demonstrated the superiority of the HeartMate III device over the HeartMate II device. This would suggest that the HeartMate III device would be superior to the HeartWare device. However, to our knowledge, there is no study comparing the continuous flow, centrifugal pump, ventricular assist devices, HeartWare and HeartMate III. Therefore, the purpose of this study is to compare HeartWare and HeartMate III LVADs and to assess whether there are significant differences in survival and adverse events between these devices.

\section{Methods}

We reviewed medical records of all adult patients who underwent VADs therapy with centrifugal pumps between 2011 and 2018 in our institution. All were in end stage heart failure. The cohort was divided into 2 subgroups according to the device implanted: HeartMate III or HeartWare. The surgeon choose the type of LVAD to implant according to his expertise. Data concerning pre and post-operative hemodynamics and biological profiles as well as complications and outcome were collected and analyzed. Primary endpoint was survival at 1 year. Secondary endpoints were device related complications. Statistical analysis was performed using SPSS BASE 12.0 statistical software (SPSS Inc. Chicago, IL, USA). Categorical variables were expressed as percentage and compared using the Chi-square or Fisher's exact test, as appropriate. Continuous variables were expressed as the mean \pm 1 standard deviation and were compared using the Mann-Whitney test for unpaired groups to avoid the assumption of normality. Survival data were analyzed with standard Kaplan-Meier actuarial techniques for estimation of survival probabilities. For survival estimates of patients

*Correspondence to: Anna Nowacka, Department of Cardiac surgery, University Hospital, Lausanne, Rue du Bugnon 46, 1011 Lausanne, Switzerland, E-mail: anna.nowacka@chuv.ch

Key words: left ventricular assist device (LVAD), HeartWare (HW), HeartMate 3 (HM3), heart failure, adverse events, survival

Received: December 11, 2019; Accepted: December 14, 2019; Published: December 18, 2019 
Nowacka A (2019) Clinical results of continuous flow ventricular assist devices: A comparative analysis between heartmate 3 and heart ware. Are centrifugal pumps all the same?

under support, patients were censored at the time of device explant because of transplantation. A two-tailed p-value less than 0.05 was taken to indicate statistical significance.

The Cantonal Medical Ethics Committee Authority approved the present study (CER-VD 2016-00820).

\section{Results}

Thirty-two (32) patients received a LVAD, 29 as bridge to transplant and 3 as destination therapy. There were 26 men and 6 female aged 55.3 \pm 10.9 years ( 25.6 to 74.4 years). The cohort was stratified into 2 groups of 16 each according to the model of LVAD they received. All the six women belong to the HeartWare group while the HM3 group gender is male $(37.5 \%$ vs. $0 \%, p=0.018)$. The two groups are not significantly different with respect to age, body area, height and weight of patients. There was significantly more ischemic heart disease in the HM3 group than in the HW group ( $75 \%$ vs. $31.3 \%, p=0.03$ ). There were no significant differences between the two groups with respect to preoperative clinical data and preoperative status (Table 1). There was only one patient in the INTERMACS 1 class (HM3 group). Patients implanted as destination therapy (3 patients) belong to the HM3 group.

There was significant difference between two groups in pulmonary arterial pressures as well as pulmonary arterial occlusion pressure (PAPO) and they were significantly higher in patients in the HM3 group than in the HW group (PAPm: $42.7 \pm 11.1$ (HM3) vs. $31.6 \pm 10.7(\mathrm{HW}), \mathrm{p}=0.014$ and PAPO: $28.8 \pm 7.4$ vs. $21.5 \pm 9.0$, $\mathrm{p}=0.03)$.

Table 1. Clinical characteristic of patients before LVAD implantation

\begin{tabular}{|c|c|c|c|c|}
\hline & All Patient (n=32) & HW (n=16) & HM3 (n=16) & $\mathbf{p}$ \\
\hline Gender (M/F) (\%) & $26 / 6(81.3 / 18.7)$ & $10 / 6(62.5 / 37.5)$ & $16 / 0(100 / 0)$ & $0.018^{*}$ \\
\hline Age (y.o) & $55.3 \pm 10.9(25.6-74.4)$ & $54.7 \pm 11.1$ & $55.8 \pm 11.10$ & 0.77 \\
\hline $\operatorname{BSA}\left(\mathrm{m}^{2}\right)$ & $1.9 \pm 0.29(1.22-2.47)$ & $1.8 \pm 0.35$ & $2.0 \pm 0.19$ & 0.06 \\
\hline Height $(\mathrm{cm})$ & $173 \pm 10.1$ & $170.3 \pm 12.3$ & $175.8 \pm 6.6$ & 0.13 \\
\hline Weight (kg) & $78.5 \pm 19.6$ & $72.3 \pm 22.6$ & $84.8 \pm 14.2$ & 0.07 \\
\hline Ischaemic heart disease & $17(53.1 \%)$ & $5(31.3 \%)$ & $12(75 \%)$ & $0.03 *$ \\
\hline Acute myocardial infarction & $6(18.8 \%)$ & $3(18.8 \%)$ & $3(18.8 \%)$ & 1.0 \\
\hline Dilated ischaemic cardiomyopathy & $11(34.3 \%)$ & $2(12.5 \%)$ & $9(56.3 \%)$ & $0.023 *$ \\
\hline Primary cardiomyopathies & $15(46.9 \%)$ & $11(68.8 \%)$ & $4(25 \%)$ & $0.03 *$ \\
\hline Idiopathic & $11(34.3 \%)$ & $8(50 \%)$ & $3(18.8 \%)$ & 0.14 \\
\hline Toxic & $1(3.1 \%)$ & $1(6.3 \%)$ & $0(0 \%)$ & NA \\
\hline Valvulopathic & $1(3.1 \%)$ & $0(0 \%)$ & $1(6.3 \%)$ & NA \\
\hline Hypertophic & $2(6.3 \%)$ & $2(12.5 \%)$ & $0(0 \%)$ & NA \\
\hline Previous cardiac surgery & $7(21.8 \%)$ & $2(12.5 \%)$ & $5(31.3 \%)$ & 0.4 \\
\hline Stroke & $7(21.8 \%)$ & $5(31.3 \%)$ & $2(12.5 \%)$ & 0.4 \\
\hline Insulin-dependent diabetes & $3(9.4 \%)$ & $1(6.3 \%)$ & $2(12.5 \%)$ & 1.0 \\
\hline Non-insulin dependent diabetes & $6(18.8 \%)$ & $1(6.3 \%)$ & $5(31.3 \%)$ & 0.17 \\
\hline Cardiac arrest $<24 \mathrm{~h}$ & $0(0 \%)$ & $0(0 \%)$ & $0(0 \%)$ & \\
\hline \multicolumn{5}{|l|}{ INTERMACS } \\
\hline $1-3$ & $15(47 \%)$ & $6(38 \%)$ & $9(56 \%)$ & 0.5 \\
\hline $4-7$ & $17(53 \%)$ & $10(63 \%)$ & $7(43.8 \%)$ & 0.5 \\
\hline \multicolumn{5}{|l|}{ Purpose of implantation } \\
\hline BTT & $29(90.6 \%)$ & $16(100 \%)$ & $13(81 \%)$ & 0.23 \\
\hline DT & $3(9.4 \%)$ & 0 & $3(18.8 \%)$ & 0.23 \\
\hline ICU stay & $8(25 \%)$ & $2(12.5 \%)$ & $6(37.5 \%)$ & 0.22 \\
\hline Preoperative mechanical ventilation $\mathrm{N}$ & $3(9.4 \%)$ & 0 & $3(18.8 \%)$ & 0.23 \\
\hline IABP & $1(3.1 \%)$ & 0 & $1(6.3 \%)$ & 1.0 \\
\hline ECLS & $1(3.1 \%)$ & 0 & $1(6.3 \%)$ & 1.0 \\
\hline \multicolumn{5}{|l|}{ Haemodynamics } \\
\hline FE (\%) & $21.3 \pm 8.2$ & $22.1 \pm 9.6$ & $20.5 \pm 6.8$ & 0.6 \\
\hline Cardiac index $\left(\mathrm{L} / \mathrm{min} / \mathrm{m}^{2}\right)$ & $2.1 \pm 0.5$ & $2.0 \pm 0.5$ & $2.3 \pm 0.4$ & 0.13 \\
\hline Central venous pressure $(\mathrm{mmHg})$ & $10.8 \pm 5.7$ & $9.4 \pm 5.8$ & $12.3 \pm 5.4$ & 0.2 \\
\hline PAPm (mmHg) & $36.5 \pm 12.0$ & $31.6 \pm 10.7$ & $42.7 \pm 11.1$ & $0.014 *$ \\
\hline \multicolumn{5}{|l|}{ Laboratory test } \\
\hline $\mathrm{Na}(\mathrm{mmol} / \mathrm{L})$ & $137.1 \pm 5.7$ & $135.4 \pm 5.2$ & $138.8 \pm 5.9$ & 0.1 \\
\hline $\mathrm{BUN}(\mathrm{mmol} / \mathrm{L})$ & $15.4 \pm 24.9$ & $18.8 \pm 35.1$ & $12.2 \pm 8.3$ & 0.5 \\
\hline Creatinine $(\mathrm{mmol} / \mathrm{L})$ & $115.8 \pm 43.4$ & $105.3 \pm 40.2$ & $126.4 \pm 45.1$ & 0.17 \\
\hline Serum total bilirubin $(\mathrm{mmol} / \mathrm{L})$ & $27.2 \pm 27.2$ & $33.6 \pm 31.5$ & $23.7 \pm 25.8$ & 0.54 \\
\hline ASAT (U/I) & $48.6 \pm 61.9$ & $55.4 \pm 81.7$ & $42.6 \pm 39.3$ & 0.6 \\
\hline ALAT (U/I) & $60.1 \pm 98.5$ & $74.8 \pm 138.9$ & $47.2 \pm 40.7$ & 0.5 \\
\hline INR & $1.2 \pm 0.36$ & $1.2 \pm 0.4$ & $1.1 \pm 0.2$ & 0.5 \\
\hline Albumin $(\mathrm{g} / \mathrm{L})$ & $36.6 \pm 7.7$ & $40.0 \pm 5.2$ & $33.5 \pm 8.5$ & $0.05 *$ \\
\hline WBC (G/L) & $8^{\prime} 132 \pm 3 ’ 274$ & 7’319 $\pm 2 ’ 759$ & 8’944 \pm 3’625 & 0.2 \\
\hline Thrombocytes (G/L) & $187^{\prime} 156 \pm 62 ’ 213$ & $219^{\prime} 500 \pm 66^{\prime} 107$ & $174^{\prime} 813 \pm 50^{\prime} 634$ & $0.04 *$ \\
\hline Hematocrit (\%) & $36.6 \pm 6.3$ & $36.8 \pm 6.5$ & $36.5 \pm 6.2$ & 0.9 \\
\hline
\end{tabular}


Nowacka A (2019) Clinical results of continuous flow ventricular assist devices: A comparative analysis between heartmate 3 and heart ware. Are centrifugal pumps all the same?

Preoperatively, the number of thrombocytes and the albumin level were significantly lower in patients in the HM3 group than in the HW group (Thrombocytes: $174^{\prime} 813 \pm 50^{\prime} 634 \mathrm{G} / \mathrm{L}(\mathrm{HM} 3)$ vs. $219^{\prime} 500 \pm$ $66 ' 107$ G/L (HW), p=0.04, albumin: $33.5 \pm 8.5 \mathrm{~g} / \mathrm{L}$ (HM3) vs. $40.0 \pm$ $5.2 \mathrm{~g} / \mathrm{L}(\mathrm{HW}), \mathrm{p}=0.05)$. The rest of the biological parameters were not significantly different between the two groups.

All devices were implanted under cardiopulmonary bypass (CPB). The time of $\mathrm{CPB}$ was significantly shorter in the HM 3 group than in the HW group (78.9 \pm 25.7 min vs. $124.4 \pm 67.9 \mathrm{~min}, \mathrm{p}=0.03)$. The number of minimally invasive procedures was significantly higher in the HW group than in the HM3 group ( $37.5 \%$ vs. $0 \%$, p=0.02).

The temporary right ventricular assistances was necessary in four patients $(12.5 \%, \mathrm{p}=0,1)$ in the HM3 group.

After surgery, anticoagulation was started within 6 to 12 hours using intravenous heparin to achieve an anti-Xa activity between 0.3 and $0.5 \mathrm{IU} / \mathrm{l}$. Aspirin was started 24hours after surgery in absence of bleeding or thrombocytopenia at a daily dose of $150 \mathrm{mg}$, lifetime.

Oral antivitamin $\mathrm{K}$ therapy was started after extubation and removal of chest drains, to maintain an INR between 2.5 and 3.5. During follow-up period one patient with HW device $(6.3 \%)$ and 11 with HM III device (68.8\%) were still under assistance. Eleven patients in the HW group $(68.8 \%)$ and three patients in the HM3 group $(18.8 \%)(\mathrm{p}=0.01)$ were transplanted with an average waiting time of $10.2 \pm 8.9$ months for the HW group and $6.8 \pm 2.2$ month for the HM3 group, $p=0.5$. The mean period under assistance was respectively $9.1 \pm 8.4$ months for HW group and $7.8 \pm 5.4$ months for HM3 group $(\mathrm{p}=0.4)$. There was no pump retrieve for recovery (bridge-to-recovery) in both groups.

Six (19\%) patients died during mechanical circulatory support, $4(25 \%)$ in the HW group, and $2(13 \%)$ in the HM3 group ( $\mathrm{p}=0.7)$; 4 deaths ( $3 \mathrm{HW}$ and $1 \mathrm{HM} 3$ ) within 6 months of implantation and 2 deaths (1 HW and $1 \mathrm{HM} 3$ ) at more than 6 months of implantation. Causes of death in both groups are listed in Table 2. During initial intensive care unit stay, deaths were mainly related with bleeding in the HW group. In the same group after ICU dismissal, deaths were related to multi organ failure or arrhythmias. In the HM3 group, we noticed two deaths related to multi organ failure (Figure 1).

The overall survival at 1,3 , and 12 months post-implantation was respectively $93.8 \% \pm 4.3 \%$ (30 patients), $87.3 \% \pm 5.9 \%$ (18 patients), ) and $79.3 \% \pm 9.3 \%$ (7 patients) in both groups combined. There were no significant differences in survival at 1,3 - and 12-months postimplantation between the two groups $(\mathrm{p}=0.75)$.

The adverse events occurring most frequently during circulatory support are listed in Table 3. Hemorrhagic complication was the most frequent in both groups $(43.8 \%$ bleeding requiring surgery or embolization) and exceed thrombotic complications (3.1\% pump thromboses and 9.4\% stroke (ischemic).

Table 2. Causes of death during mechanical circulatory support

\begin{tabular}{|c|c|c|c|}
\hline & All & HW & HM 3 \\
\hline In ICU death & & & 0 \\
\hline Bleeding & 2 & $2(0-0,5$ months $)$ & $1(2.48$ months $)$ \\
\hline Multi organ failure & 2 & $1(1.12$ months $)$ & \\
\hline Post ICU death & & & $1(9.04$ months $))$ \\
\hline Respiratory failure & 1 & 0 & 0 \\
\hline Arrhythmias & 1 & $1(18.8$ months $)$ & \\
\hline
\end{tabular}

Table 3. Adverse events observed during mechanical circulatory support.

\begin{tabular}{|l|c|c|c|c|}
\hline \multicolumn{1}{|c|}{ All } & HW & HM 3 & p \\
\hline Bleeding and cardiac tamponade & $\begin{array}{c}14 \\
(43.8 \%)\end{array}$ & $9(56.3 \%)$ & $5(31.3 \%)$ & 0.29 \\
\hline $\begin{array}{l}\text { Gastro-intestinal or nasopharyngeal } \\
\text { bleeding }\end{array}$ & $7(21.9 \%)$ & $3(18.8 \%)$ & $4(25 \%)$ & 1.0 \\
\hline RV dysfunction & $6(18.8 \%)$ & $2(12.5 \%)$ & $4(25 \%)$ & 0.65 \\
\hline Pump thrombosis & $1(3.1 \%)$ & $1(6.3 \%)$ & 0 & 1.0 \\
\hline Pump exchange & $1(3.1 \%)$ & $1(6.3 \%)$ & 0 & 1.0 \\
\hline Device dysfunction & 0 & 0 & 0 & NA \\
\hline Stroke & $3(9.4 \%)$ & $2(12.5 \%)$ & $1(6.3 \%)$ & 1.0 \\
\hline Transient ischemic attack & $1(3.1 \%)$ & $0(0 \%)$ & $1(6.3 \%)$ & 1.0 \\
\hline Driveline infection & $2(6.3 \%)$ & $1(6.3 \%)$ & $1(6.3 \%)$ & 1.0 \\
\hline Pump infection & $1(3.1 \%)$ & 0 & $1(6.3 \%)$ & 1.0 \\
\hline
\end{tabular}

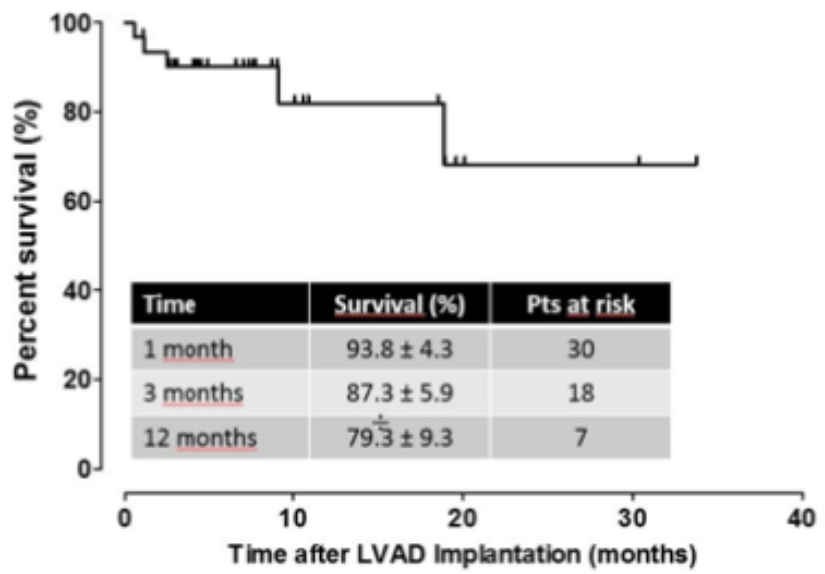

A

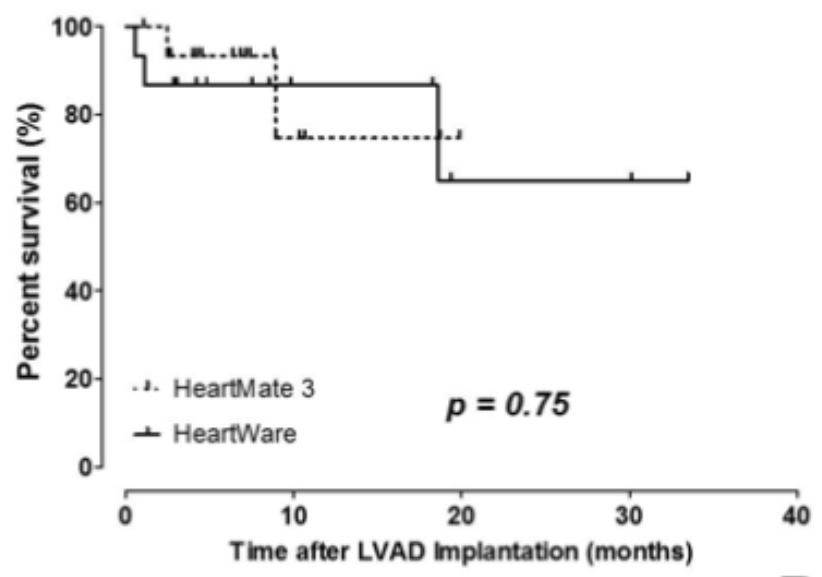

B

Figure 1. Actuarial estimates of survival under mechanical circulatory support in (A) the whole patient population $(\mathrm{N}=32)$ and in $(\mathrm{B})$ patients under $\mathrm{HW}(\mathrm{N}=16)$ and $\mathrm{HM} 3(\mathrm{~N}=16)$ support

There was only one pump thrombosis (HW group) which also required a change of the dispositive. No HM3 device had mechanical dysfunction.

For the right ventricular dysfunction, the two temporary right ventricular assistances of the HW group were implanted in the postoperative period, while all those in the HM3 group were implanted intraoperatively. 
Nowacka A (2019) Clinical results of continuous flow ventricular assist devices: A comparative analysis between heartmate 3 and heart ware. Are centrifugal pumps all the same?

Post-transplant follow-up was significantly higher in the HW group than in the HM3 group $(37.9 \pm 18.5$ months (HW) vs. $8.0 \pm 3.5$ months (HM 3), $\mathrm{p}=0.0001$ ).

\section{Discussion}

We have experienced remarkable progress in mechanical circulatory support devices for patients in end-stage heart failure: there is a clear improvement of patient's functional capacity, quality of life and survival when a centrifugal LVAD is implanted. Long-term mechanical circulatory support avoids also deterioration of secondary organs due to low cardiac output and therefore increases the chances of successful heart transplant.

A randomized study of Slaughter et al. demonstrated the superiority in survival, durability, and decrease in the number of adverse events of the second-generation continuous flow VAD (HeartMate II) compared to pulsatile flow.

Although improving the survival and quality of life of patients with end-stage heart failure, LVAD implantation is also associated with a number of complications. Among the most common and the most feared are hemorrhages, infections, stroke, right ventricular dysfunction and pump dysfunction [5-9,10].

Bleeding is the most common reported complication associated with LVAD implantation. Hemorrhage can occur in the perioperative period or during the longest course, favored by the necessary of anticoagulation under assistance, the development of an acquired von Willebrand disease and finally lost of pulsatility under continuous flow assistance. The gastrointestinal tract is the site of choice for bleeding more than 3 months after implantation [9]. The rate of bleeding reported in the literature is $33-44 \%$ for HM3 device, of which $10-16 \%$ require surgery For the HW device, bleeding rates requiring reoperation are $13-20 \%[5,6]$.

As expected, bleeding was the most common complication in both groups: $43.8 \%$ of patients (56.3\% HW and $31.3 \% \mathrm{HM} 3$ ) requiring surgery or embolization, of which $21.9 \%$ were gastrointestinal or nasopharyngeal bleeding. The higher surgical intervention rates in our study may be explained by addition of all surgical procedures (e.g. nasopharyngeal cauterization, chest drainage included as interventional procedure)

The second complication in terms of frequency in the first 3 months post-implantation are infections: pump, cannula or pocket infections, and finally, most frequent, driveline infections.

Considering driveline infections $(6.3 \%$ overall, $6.3 \%$ (HW) vs. $6.3 \%$ (HM3), $\mathrm{p}=1.0$ ), we have reported infections requiring surgical intervention and not those treated with antibiotics and local care which may explain lower rates than in the literature (6 to $34 \%$ for HW [7] and 12 to $16 \%$ for HM3 $[5,6]$.

Ischemic stroke and intracranial hemorrhage following VAD placement are another major cause of morbidity. They represent a major cause of mortality with a predominance in the first 3 months after implantation.

We report stroke rates at $9.4 \%$ in total, $12.5 \%$ (HW) and $6.3 \%$ (HM3), ( $\mathrm{p}=1.0)$ which was not significantly different between the two groups, contrary to recent data in the literature reporting higher rates of stroke with the HW device. [4] In addition, these rates are comparable to or even lower than the literature data ( $8 \%$ to $29.7 \%$ for HW $[4,7,11]$ and $8 \%$ to $18 \%$ for HM $3[5,6]$. In our study, all the strokes are ischemic.
The development or deterioration of right heart failure under LVAD is an important cause of morbidity and mortality, especially in the first 3 months after implantation. When a LVAD discharges the left ventricle, which restored a normal cardiac output and can leads to the right ventricle dysfunction (often an underlying bi-ventricular dysfunction is present). On the other hand, LVADs tend to decrease the pressures and volume of the left ventricle, which can alter the geometry and function of the right ventricle.

In our study, right ventricular dysfunction requiring temporary right ventricular assist is the second most common complication in both groups (18.8\% of patients in both groups, $12.5 \%$ (HW) vs. $25 \%$ (HM3), $\mathrm{p}=0.65)$. In the literature, this complication is less frequent and occurs in $2.6 \%$ to $4 \%$ patients with a HM3 $[5,6]$ and $2 \%$ to $6 \% \mathrm{HW}$ patients [7]. A more liberal implantation of right ventricular support perioperatively, may possibly explain the higher rates of temporary right ventricular assistance in the HM3 group compared to the literature.

Pump thrombosis rates requiring a change in assistance $(3.1 \%$ of all patients, $6.3 \%$ of $\mathrm{HW}$ vs. $0 \%$ of $\mathrm{HM} 3 \mathrm{p}=1.0$ ) are comparable to those in the literature $2 \%$ to $8 \%$ [7] HW and $0 \%[5,6]$ of HM3.

According to the 8th annual INTERMACS report published in 2017, the overall survival with or without concomitant right ventricular assist is $95 \%, 81 \%$ and $70 \%$ respectively at 1 month, 12 months and 24 months post implantation [9]. This report includes data from 17,634 patients implanted with a continuous flow ventricular assist device (17,016 LVAD and 618 BiDAV) between 2008 and 2016. For centrifugal continuous flow devices, the literature reports survival rates of $87-94 \%$ at 6 months and $84-86 \%$ at 12 months for the HW [7], 98\%, 92\% and $81 \%$ respectively at 1, 6 and 12 months for the HM3 [6].

The overall survival rates in our study at 1,3 and 12 months postimplantation in the group of HW $87.5 \% \pm 8.3 \%, 81.3 \% \pm 9.8 \%$ and $81.3 \% \pm 9.8 \%$ and HM3 $93.3 \% \pm 6.4 \% ; 93.3 \% \pm 6.4 \%$ and $74.7 \% \pm 17.5 \%$ are comparable to the survival rates found in the literature

Unfortunately, the 12-month survival rate in the HM3 group affects only 4 patients. This is explained by short time of follow-up in view of the recent availability of the HM3 device.

\section{Limits}

An important limitation of this study is its retrospective design. In fact, despite careful research, post-implantation adverse events could be missed, either because they were not reported in the files or because of data collection deficiencies.

Another limitation of this study is a follow-up bias for the HM3 group. Seven patients in the HeartMate III group do not reach the 12-month follow-up. The difference false the interpretation of the postoperative results, more particularly concerning the undesirable events.

\section{Conclusions}

Our experience showed that HM3 and HV devices are equivalent with respect to survival, intraoperative features and major complications. Both devices provided excellent hemodynamic support, had similar stroke and bleeding complication rates. Both devices can be safety used for circulatory support with similar survival rates.

\section{References}

1. Mosterd A, Hoes AW (2007) Clinical epidemiology of heart failure. Heart 93: 11371146. [Crossref] 
2. van Riet EE, Hoes AW, Wagenaar KP, Limburg A, Landman MA, et al. (2016) Epidemiology of heart failure: The prevalence of heart failure and ventricular dysfunction in older adults over time. A systematic review. Eur J Heart Fail 18: 242252. [Crossref]

3. Heidenreich PA, Albert NM, Allen LA, Bluemke DA, Butler J, et al. (2013) Forecasting the impact of heart failure in the United States: A policy statement from the American Heart Association. Circ Heart Fail 6: 606-619. [Crossref]

4. Rogers JG, Pagani FD, Tatooles AJ, Bhat G, Slaughter MS, et al, (2017) Intrapericardial Left Ventricular Assist Device for Advanced Heart Failure. N Engl J Med 376: 451-460. [Crossref]

5. Mehra MR, Naka Y, Uriel N, Goldstein DJ, Cleveland JC Jr, et al. (2016) A Fully Magnetically Levitated Circulatory Pump for Advanced Heart Failure. N Engl J Med 376: 440-450. [Crossref]

6. Krabatsch T, Netuka I, Schmitto JD, Zimpfer D, Garbade J, et al. (2017) Heartmate 3 fully magnetically levitated left ventricular assist device for the treatment of advanced heart failure -1-year results from the Ce mark trial. J Cardiothoracic Surg 12: 23.
7. Pozzi M, Giraud R, Tozzi P, Bendjelid K, Robin J, et al. (2015) Long-term continuous flow left ventricular assist devices (LVAD) as bridge to heart transplantation. J Thorac Dis 7: 532-542. [Crossref]

8. Slaughter MS, Rogers JG, Milano CA, Russell SD, Conte JV, et al. (2009) Advanced Heart Failure Treated with Continuous-Flow Left Ventricular Assist Device. N Engl J Med 361: 2241-2251. [Crossref]

9. Kirklin JK, Pagani FD, Kormos RL, Stevenson LW, Blume ED, et al. (2017) Eighth annual INTERMACS report: Special focus on framing the impact of adverse events. J Heart Lung Transplant 36: 1080-1086. [Crossref]

10. Ponikowski P, Voors AA, Anker SD, Bueno H, Cleland JG, et al. (2016) 2016 ESC Guidelines for the diagnosis and treatment of acute and chronic heart failure: The Task Force for the diagnosis and treatment of acute and chronic heart failure of the European Society of Cardiology (ESC)Developed with the special contribution of the Heart Failure Association (HFA) of the ESC. Eur J Heart Fail 37: 2129-2200. [Crossref]

11. Cho SM, Moazami N, Frontera JA (2017) Stroke and Intracranial Hemorrhage in HeartMate II and HeartWare Left Ventricular Assist Devices: A Systematic Review. Neurocrit Care 27: 17-25. [Crossref]

Copyright: (C2019 Nowacka A. This is an open-access article distributed under the terms of the Creative Commons Attribution License, which permits unrestricted use, distribution, and reproduction in any medium, provided the original author and source are credited. 